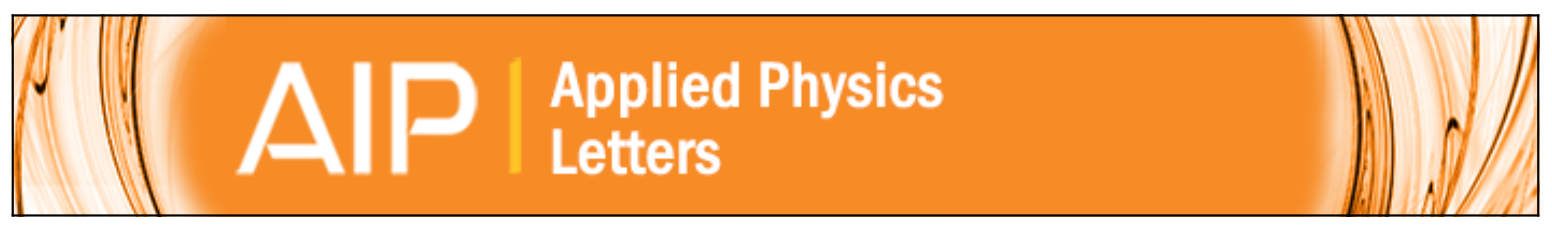

\title{
Whispering gallery modes in indium oxide hexagonal microcavities
}

Hongxing Dong, Zhanghai Chen, Liaoxin Sun, Jian Lu, Wei Xie, H. Hoe Tan, Chennupati Jagadish, and Xuechu Shen

Citation: Applied Physics Letters 94, 173115 (2009); doi: 10.1063/1.3126443

View online: http://dx.doi.org/10.1063/1.3126443

View Table of Contents: http://scitation.aip.org/content/aip/journal/apl/94/17?ver=pdfcov

Published by the AIP Publishing

\section{Articles you may be interested in}

Infrared light detection using a whispering-gallery-mode optical microcavity

Appl. Phys. Lett. 104, 171114 (2014); 10.1063/1.4874652

Tunable add-drop filter using an active whispering gallery mode microcavity

Appl. Phys. Lett. 103, 181103 (2013); 10.1063/1.4827637

Determination of binding kinetics using whispering gallery mode microcavities

Appl. Phys. Lett. 99, 103703 (2011); 10.1063/1.3634023

Combined whispering gallery mode laser from hexagonal $\mathrm{ZnO}$ microcavities Appl. Phys. Lett. 95, 191117 (2009); 10.1063/1.3264080

Whispering gallery modes in hollow cylindrical microcavities containing silicon nanocrystals Appl. Phys. Lett. 92, 131119 (2008); 10.1063/1.2903134

\section{AIP $\mid$ APL Photonics}

APL Photonics is pleased to announce Benjamin Eggleton as its Editor-in-Chief 


\title{
Whispering gallery modes in indium oxide hexagonal microcavities
}

\author{
Hongxing Dong, ${ }^{1}$ Zhanghai Chen, ${ }^{1, a)}$ Liaoxin Sun, ${ }^{1}$ Jian Lu, ${ }^{1}$ Wei Xie, ${ }^{1}$ H. Hoe Tan, ${ }^{2}$ \\ Chennupati Jagadish, ${ }^{2}$ and Xuechu Shen ${ }^{1}$ \\ ${ }^{1}$ Department of Physics, Surface Physics Laboratory, Advanced Materials Laboratory, Fudan University, \\ Shanghai 200433, China \\ ${ }^{2}$ Department of Electronic Materials Engineering, Research School of Physics and Engineering, \\ The Australian National University, Canberra, Australian Capital Territory 0200, Australia
}

(Received 19 January 2009; accepted 2 April 2009; published online 1 May 2009)

\begin{abstract}
We report on the use of $\operatorname{In}_{2} \mathrm{O}_{3}$ nanowires with hexagonal cross section as optical whispering gallery resonators. The single-crystal $\mathrm{In}_{2} \mathrm{O}_{3}$ nanowires were fabricated by an in situ thermal oxidation method. Whispering gallery modes (WGMs) in the visible spectral range were directly observed at room temperature. Due to the slight tapering of the nanowires, the energies and orders of the WGMs were modulated when excitations were scanned along the $c$-axis (length) of the nanowires. The experimental results were explained and fitted well with a plane wave interference model and Cauchy dispersion formula for refractive indices. (C) 2009 American Institute of Physics. [DOI: $10.1063 / 1.3126443$ ]
\end{abstract}

Semiconductor microcavities have been investigated and used widely in optoelectronics; among them, whispering gallery (WG) resonators have attracted much attention due to their microscopic size, high quality factor $Q$, and low lasing threshold power. $^{1-3}$ In a semiconductor WG resonator, the nanostructure itself functions as both the gain medium and the optical microcavity. Light wave can be mostly confined inside the microcavity due to multiple total internal reflections (TIRs) at the cavity boundary. The electromagnetic field and the photon density of states can be precisely controlled in a prescribed manner. Such a precise manipulation of light-matter interaction is essential for various purposes, ranging from the fundamental studies on quantum electrodynamics to the development of miniature optoelectronic devices, including lasers, ${ }^{4}$ optical waveguides, ${ }^{5}$ tunable filters, ${ }^{6}$ and optical sensors. ${ }^{7}$ It is known that WG resonators with different geometries, such as rings, ${ }^{8}$ microspheres, ${ }^{9}$ cylinders, ${ }^{10}$ nanonails, ${ }^{11}$ and nanowires with hexagonal cross section, 2,4,12 have been developed. Among them, micro-/ nanowires with hexagonal cross section have attracted particular interest due to their better optical properties and wider range of potential applications. However, the investigation is so far limited to $\mathrm{ZnO}$ hexagonal resonators due to the difficulty in synthesizing high quality hexagonal microcavities with other materials.

Indium oxide is one of the important oxide materials in optoelectronics and widely studied in recent years. Welldefined $\mathrm{In}_{2} \mathrm{O}_{3}$ nanostructures with different morphologies, especially one-dimensional $\mathrm{In}_{2} \mathrm{O}_{3}$ nanowires as building blocks for photonic devices have been fabricated by various methods. ${ }^{13-15} \mathrm{Up}$ to now, most of the synthesized $\mathrm{In}_{2} \mathrm{O}_{3}$ nanowires have rectangular cross sections or irregular circular cross sections with rough surfaces. In addition, the lateral size of these nanowires is typically too small to be used as a WG mode cavity. As a result, the application of $\mathrm{In}_{2} \mathrm{O}_{3}$ nanowires as optical cavities has not yet been explored so far. In this letter, we report the fabrication of high quality $\operatorname{In}_{2} \mathrm{O}_{3}$ nanowires with hexagonal cross section by an in situ thermal

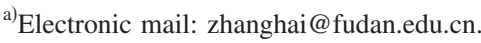

oxidation method and the observation of a series of WGM resonances in these wires. Tapering of these nanowires enables the wavelength of cavity modes and the order of resonance to be modulated continuously. The modulation was observed at room temperature and mapped directly by using microphotoluminescence spectroscopy. Calculations based on a simple plane wave interference model and Cauchy dispersion functions agree well with the experimental data.

The $\mathrm{In}_{2} \mathrm{O}_{3}$ nanowires were fabricated by a simple thermal oxidation of indium metal in a conventional horizontal tube furnace using $\mathrm{H}_{2} \mathrm{O}$ vapor as the oxide source material. Prior to the synthesis, indium grains (purity of 99.999\%) with diameter of 2-3 mm were treated in an aqueous $1.0 \mathrm{M}$ $\mathrm{HCl}$ solution for $30 \mathrm{~s}$, and then cleaned with absolute ethanol in an ultrasonic bath for $5 \mathrm{~min}$. After they had been coated with a layer of Au catalyst, the indium grains were placed in a quartz boat. The boat was then positioned in the middle of the quartz tube which was located in the middle of the furnace. Before heating, high-purity $\mathrm{N}_{2}(99.999 \%)$ was introduced into the quartz tube to purge the air inside. After 30 min of purging, the system was then heated to $800{ }^{\circ} \mathrm{C}$ over 40 min with a constant flow of $\mathrm{N}_{2}$ gas at a rate of $1.0 \mathrm{~L} / \mathrm{h}$. Afterwards, $\mathrm{H}_{2} \mathrm{O}$ vapor was introduced into the tube by bubbling $\mathrm{N}_{2}$ over a beaker of water at a flow of $2.0 \mathrm{~L} / \mathrm{h}$. The temperature was kept at $800{ }^{\circ} \mathrm{C}$ for $60 \mathrm{~min}$. After the reaction had finished, the system was cooled down to room temperature with a constant flow of $\mathrm{N}_{2}$ gas at a rate of $1.0 \mathrm{~L} / \mathrm{h}$.

The samples were first characterized by scanning electron microscopy (SEM) and transmission electron microscopy (TEM). Figure 1(a) shows the SEM image of a typical single $\mathrm{In}_{2} \mathrm{O}_{3}$ nanowire, which has been removed from the indium host and dispersed on a piece of silicon wafer. The nanowire is slightly tapered in shape with microscale diameters. Higher magnification image reveals that the cross section of the nanowire is hexagonal, as shown in the inset of Fig. 1(a). TEM observation further reveals that the $\mathrm{In}_{2} \mathrm{O}_{3}$ nanowire has a very uniform morphology and smooth surface [Fig. 1(b)]. Energy dispersive X-ray (EDX) spectroscopy data [Fig. 1(c)] indicates that the $\operatorname{In}_{2} \mathrm{O}_{3}$ is indeed stoichiometric in composition. The $\mathrm{Cu}$ and $\mathrm{C}$ signals in the EDX spectrum come from the grid used for TEM measurements. 

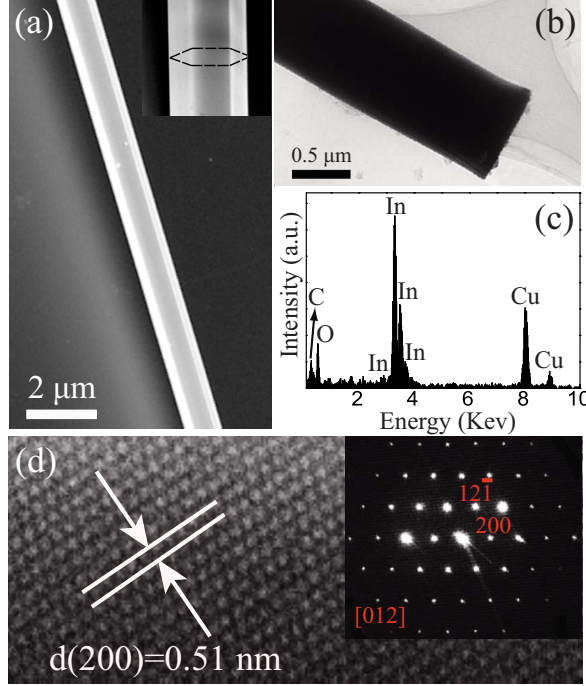

FIG. 1. (Color online) (a) SEM image of a typical $\operatorname{In}_{2} \mathrm{O}_{3}$ nanowire. The nanowire is slightly tapered. High resolution SEM (inset) indicating the hexagonal cross section of the nanowire. (b) TEM image of the $\mathrm{In}_{2} \mathrm{O}_{3}$ nanowire showing smooth surfaces. (c) EDX spectroscopy indicates a stoichiometric $\mathrm{In}_{2} \mathrm{O}_{3}$ nanowire. (d) HRTEM image and the corresponding SAED pattern (inset) indicate that the $\operatorname{In}_{2} \mathrm{O}_{3}$ nanowire is single-crystal with [100] as the growth direction.

The high-resolution TEM (HRTEM) image and the corresponding selected area electron diffraction (SAED) pattern [inset in Fig. 1(d)] indicate that the $\operatorname{In}_{2} \mathrm{O}_{3}$ nanowire is single crystal with growth proceeding along the [100] direction. The above results indicate that our $\operatorname{In}_{2} \mathrm{O}_{3}$ nanowires are of good crystalline quality with hexagonal cross section and smooth surfaces. These properties make $\operatorname{In}_{2} \mathrm{O}_{3}$ nanowires a new and exciting candidate for optical microcavity studies. The results from optical spectroscopic experiments on these tapered $\operatorname{In}_{2} \mathrm{O}_{3}$ nanowires are presented in the following section.

The nanowires used for the spectroscopic measurements have flat and smooth hexagonal resonator facets, as shown in Fig. 2(a). From data plotted in Fig. 2(b), it can be seen that the diameter of the nanowire decreases continuously along the length of the nanowire. The wavelength of the WGMs and the order of the resonance can be tuned and controlled by the diameter of the resonator. The inset of Fig. 2(b) depicts schematically a nanowire with hexagonal cross section where light wave can circle around the cavity due to TIRs at
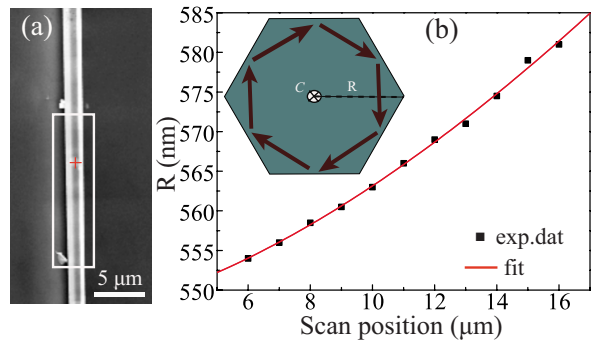

FIG. 2. (Color online) (a) SEM image of the nanowire and the focused laser spot is marked by a cross. The white rectangle is the region investigated in this work. (b) The measured cavity radius $R$ along the boxed region (data points) shows that the nanowire is slightly tapered along its length. The experimental data determined by SEM with an accuracy of $20 \mathrm{~nm}$ fitted by $R=547+0.4128 x^{1.597}$ (solid curve). The inset is a schematic of a nanowire with hexagonal cross section, showing light being confined in the WGM resenator due to TIR as inclicated by the arrows.

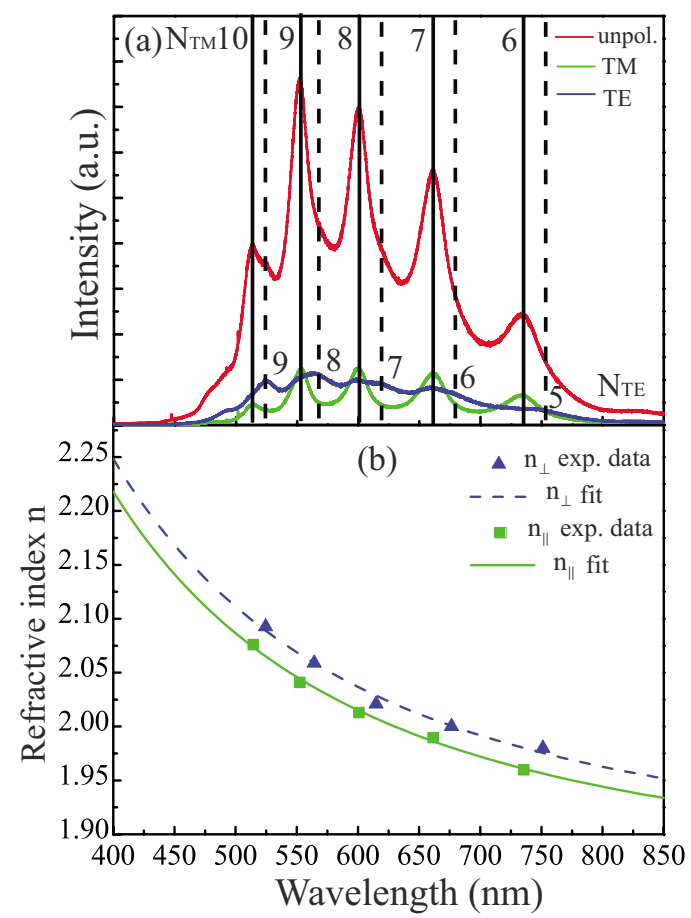

FIG. 3. (Color online) (a) The WGM photoluminescence spectra for unpolarized (red curve), TM-polarized (green curve) and TE-polarized (blue curve) light in the visible region. The two sets of integers are the interference order for the corresponding resonant mode peaks. (b) The wavelengthdependent refractive indices of the $\mathrm{In}_{2} \mathrm{O}_{3}$ nanowires $n_{\|}$(TM mode) and $n_{\perp}$ (TE mode), were deduced by fitting the scattered peak-position refractive indices to Cauchy dispersion functions.

the interface between nanowire and air, hence forming a WGM. The flat and smooth surface morphology is crucial for the waveguiding performance without large losses from the surfaces, thereby leading to a high quality factor and low lasing threshold of the cavity.

Optical studies of individual $\operatorname{In}_{2} \mathrm{O}_{3}$ nanowires were carried out with a confocal microphotoluminescence spectrometer. A He-Cd laser with an emission wavelength of $325 \mathrm{~nm}$ was used as the excitation light source. The laser beam was focused on the sample by a UV microscopic objective (40×), to a spot diameter of about $1 \mu \mathrm{m}$. We performed photoluminescence measurements with detection of unpolarized, TM-polarized (the electrical component of light $E \|_{c \text {-axis), }}$ and TE-polarized ( $E \perp c$-axis) signals. Figure 3(a) shows the typical spectra of the $\operatorname{In}_{2} \mathrm{O}_{3}$ nanowire in the visible region. The laser spot was focused at the center of the nanowire, as shown by the cross in Fig. 2(a). The physical origin of visible emission is mainly due to oxygen related defects or indium vacancies. ${ }^{16,17}$ From Fig. 3(a), we can see that the unpolarized signal is dominated by the TM component. The TE modes are weak and broad. These results are similar to the previous investigations of WGMs in hexagonal $\mathrm{ZnO}$ optical cavities. $^{2,12}$ Thus, the losses in the cavity are different for both TE and TM polarization, with TM modes having lower losses.

To further explore the characteristics of the $\mathrm{In}_{2} \mathrm{O}_{3}$ nanowire WGMs, a simple plane wave model was used to fit the experimental results. The radius of the resonator $R$, as shown in Fig. 2(b), can be written as 


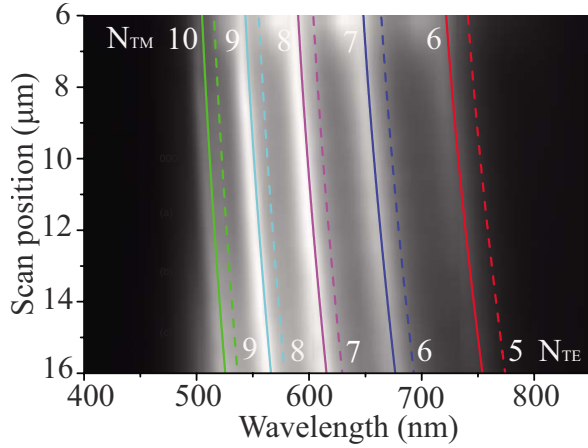

FIG. 4. (Color online) Spatially resolved photoluminescence mapping of the $\mathrm{In}_{2} \mathrm{O}_{3}$ nanowire along its length (unpolarized detection). A redshift in the spectral maxima was detected with the WGM microcavity radius. The colored solid curves (TM mode) and dashed curves (TE mode) are theoretical fittings based on a plane wave interference model. The fittings are in good agreement with the experiment results.

$$
R=\frac{h c}{3 \sqrt{3} n E}\left[N+\frac{6}{\pi} \arctan \left(\beta \sqrt{3 n^{2}-4}\right)\right],
$$

where $h$ is Planck's constant, $c$ is the speed of light in vacuum, $N$ is the interference order, and $n$ is the refractive index. The factor $\beta$ denotes polarization, for TM polarization $\beta=n^{-1}$, and for TE polarization $\beta=n$. Both TM and TE modes were used for the theoretical fitting, and the calculated results are shown in Fig. 3(a).

Taking the refractive index $n$ of $\operatorname{In}_{2} \mathrm{O}_{3}$ to be 2 for the visible spectral range, ${ }^{18}$ and the radius of the hexagonal cavity $R$ as $566 \mathrm{~nm}$ (as measured by SEM) at the measurement point on the nanowire, we were able to initially identify the interference order $N$ for the TM and TE modes. However, we found that the results are not in good agreement with the experimental peaks when a constant value of $n=2$ was used. This is most likely due to the fact that wavelength dependence of the refractive index is not taken into account. To obtain accurate values of $n_{\|}$and $n_{\perp} \quad\left(n_{\|}\right.$and $n_{\perp}$ being the refractive indices for the TM and TE modes, respectively) for the $\mathrm{In}_{2} \mathrm{O}_{3}$ nanowire at the WGM-peak wavelengths, the interference order, $N_{\mathrm{TM}}=6-10, N_{\mathrm{TE}}=5-9$, and the cavity radius $R=566 \mathrm{~nm}$ were used in the WGM equation. The obtained scattered wavelength-dependent refractive indices of the $\mathrm{In}_{2} \mathrm{O}_{3}$ nanowire $n_{\|}$and $n_{\perp}$ were fitted by using Cauchy dispersion formula as follows:

$$
n_{\|}=1.8529+\frac{59220.04}{\lambda^{2}}, \quad n_{\perp}=1.8591+\frac{64257.61}{\lambda^{2}} .
$$

The refractive indices obtained from the fitting procedure are shown in Fig. 3(b). We can see that the refractive index dispersion is slightly different for $n_{\perp}$ and $n_{\|}\left(n_{\perp}\right.$ is larger than $n_{\|}$at the same wavelength), which is most likely attributed to the cubic phase of $\operatorname{In}_{2} \mathrm{O}_{3}$ and the anisotropic strain introduced during the crystal growth process. ${ }^{19}$

To further investigate the wavelength-dependent refractive indices of the $\operatorname{In}_{2} \mathrm{O}_{3}$ nanowire shown in Fig. 3(b), and also to investigate the modulation of WGMs with varying microcavity radius, we scanned the laser in steps of $0.5 \mu \mathrm{m}$ along the $c$-axis of nanowire, as shown by the rectangular box in Fig. 2(a), while collecting unpolarized emission from the nanowire. From the results shown in Fig. 4, we see that the visible luminescence band is clearly modulated as the excitation laser is scanned along the nanowire axis. The spectral maxima are redshifted as the radius of the hexagonal WGM cavity increases. Theoretical analysis by using the plane wave interference model was applied to explain the measured resonance energies of the WGMs, using the experimentally extracted $n_{\|}$and $n_{\perp}$ of Fig. 3(b) to calculate the resonance energies. The calculated results are shown with solid and dash lines in Fig. 4 for the TM and TE modes, respectively. It is obvious that the calculated cavity modes fit well with the measured resonances, confirming the validity of the wavelength-dependent refractive indices of the $\mathrm{In}_{2} \mathrm{O}_{3}$ nanowire, fitted by using Cauchy dispersion formula.

In summary, we have reported the synthesis of indium oxide nanowires with hexagonal cross section and the WGM resonator characteristics of these wires. SEM and TEM studies show the hexagonal single-crystal $\mathrm{In}_{2} \mathrm{O}_{3}$ nanowires have very smooth surfaces and highly uniform morphology and can be used as optical cavities. WGMs in the visible spectral range were directly observed at room temperature by using the microphotoluminescence technique. We find that the $\mathrm{In}_{2} \mathrm{O}_{3}$ nanowire WGMs are preferentially TM polarized and the WGM-peak positions can be calculated using a simple plane wave interference model and Cauchy dispersion equation. The results indicate that hexagonal $\operatorname{In}_{2} \mathrm{O}_{3}$ nanowires are suitable for forming WGM optical microcavities in the visible light spectral region.

The work is funded by the NSFC 973 projects and STCSM of China (Grant Nos. 2004CB619004 and 2006CB921506). The authors thank the Australian Government Department of Innovation, Industry, Science and Research for funding this collaborative research under the International Science Linkages (China) Program.

${ }^{1}$ A. C. Tamboli, E. D. Haberer, R. Sharma, K. H. Lee, S. Nakamura, and E. L. Hu, Nat. Photonics 1, 61 (2007).

${ }^{2}$ T. Nobis, E. M. Kaidashev, A. Rahm, M. Lorenz, and M. Grundmann, Phys. Rev. Lett. 93, 103903 (2004).

${ }^{3}$ F. Qian, Y. Li, S. Gradecak, H.-G. Park, Y. Dong, Y. Ding, Z. L. Wang, and C. M. Lieber, Nature Mater. 7, 701 (2008).

${ }^{4}$ C. Czekalla, C. Sturm, R. Schmidt-Grund, B. Q. Cao, M. Lorenz, and M. Grundmann, Appl. Phys. Lett. 92, 241102 (2008).

${ }^{5}$ V. N. Astratov, J. P. Franchak, and S. P. Ashili, Appl. Phys. Lett. 85, 5508 (2004).

${ }^{6}$ L. Maleki, A. A. Savchenko, A. B. Matsko, and V. S. Ilchenko, Proc. SPIE 5435, 178 (2004).

${ }^{7}$ A. Francois and M. Himmelhaus, Appl. Phys. Lett. 92, 141107 (2008).

${ }^{8}$ D. K. Armani, T. J. Kippenberg, S. M. Spillane, and K. J. Vahala, Nature (London) 421, 925 (2003).

${ }^{9}$ M. V. Artemyev, U. Woggon, R. Wannemacher, H. Jaschinski, and W. Langbein, Nano Lett. 1, 309 (2001).

${ }^{10}$ T. Nobis and M. Grundmann, Phys. Rev. A 72, 063806 (2005).

${ }^{11}$ J. Z. Liu, S. Lee, Y. H. Ahn, J. Y. Park, K. H. Koh, and K. H. Park, Appl Phys. Lett. 92, 263102 (2008).

${ }^{12}$ L. X. Sun, Z. H. Chen, Q. J. Ren, K. Yu, L. H. Bai, W. H. Zhou, H. Xiong, Z. Q. Zhu, and X. C. Shen, Phys. Rev. Lett. 100, 156403 (2008).

${ }^{13}$ C. H. Liang, G. W. Meng, Y. Lei, F. Phillipp, and L. D. Zhang, Adv. Mater. (Weinheim, Ger.) 13, 1330 (2001).

${ }^{14}$ J. Y. Lao, J. Y. Huang, D. Z. Wang, and Z. F. Ren, Adv. Mater. (Weinheim, Ger.) 16, 65 (2004).

${ }^{15}$ A. Vomiero, S. Bianchi, E. Comini, G. Faglia, M. Ferroni, and G. Sherveglieri, Cryst. Growth Des. 7, 2500 (2007).

${ }^{16}$ Y. B. Li, Y. Bando, and D. Golberg, Adv. Mater. (Weinheim, Ger.) 15, 581 (2003).

${ }^{17}$ M. R. Shi, F. Xu, K. Yu, Z. Q. Zhu, and J. H. Fang, J. Phys. Chem. C 111 16267 (2007).

${ }^{18}$ T. Tsuruoka, C. H. Liang, K. Terabe, and T. Hasegawa, J. Opt. A, Pure Appl. Opt. 10, 055201 (2008).

${ }^{19}$ E. Hecht, OPTICS 3rd ed. (Addison-Wesley, Boston, 1998), p. 331 